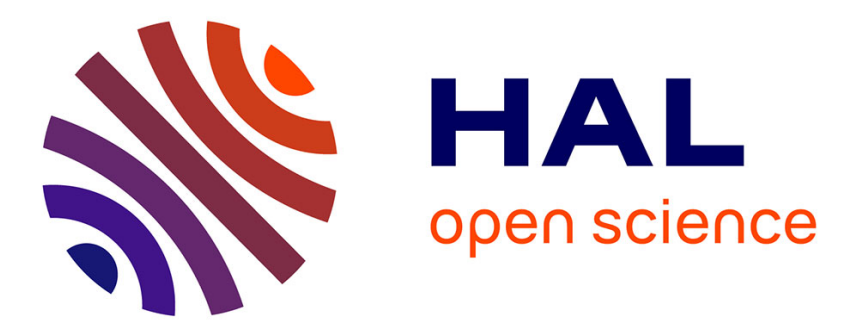

\title{
Peer-to-Peer Control for Networked Microgrids: Multi-Layer and Multi-Agent Architecture Design
}

Yu Wang, Tung-Lam Nguyen, Yan Xu, Quoc-Tuan Tran, Raphael Caire

\section{To cite this version:}

Yu Wang, Tung-Lam Nguyen, Yan Xu, Quoc-Tuan Tran, Raphael Caire. Peer-to-Peer Control for Networked Microgrids: Multi-Layer and Multi-Agent Architecture Design. IEEE Transactions on Smart Grid, 2020, 11 (6), pp.4688-4699. 10.1109/TSG.2020.3006883 . hal-03260233

\section{HAL Id: hal-03260233 https://hal.science/hal-03260233}

Submitted on 20 Jan 2022

HAL is a multi-disciplinary open access archive for the deposit and dissemination of scientific research documents, whether they are published or not. The documents may come from teaching and research institutions in France or abroad, or from public or private research centers.
L'archive ouverte pluridisciplinaire HAL, est destinée au dépôt et à la diffusion de documents scientifiques de niveau recherche, publiés ou non, émanant des établissements d'enseignement et de recherche français ou étrangers, des laboratoires publics ou privés. 


\title{
Peer-to-Peer Control for Networked Microgrids: Multi-Layer and Multi-Agent Architecture Design
}

\author{
Yu Wang, Member, IEEE, Tung-Lam Nguyen, Yan Xu, Senior Member, IEEE, Quoc-Tuan Tran, Senior \\ Member, IEEE, Raphael Caire, Senior Member, IEEE.
}

\begin{abstract}
The increasing integration of microgrids (MGs) in distribution networks forms the networked microgrids (NMGs). The peer-to-peer (P2P) control architecture is able to fully exploit the flexibility and resilience of NMGs. This paper proposes a multi-layer and multi-agent architecture to achieve P2P control of NMGs. The control framework is fully distributed and contains three control layers operated in the agent of each MG. For primary control, a droop control is adopted by each MG-agent for localized power sharing. For secondary control , a distributed consensus algorithm is proposed for frequency/voltage restoration and arbitrary power sharing among MGs. For tertiary control, the power loss in the NMG system is minimized by using alternating direction method of multipliers (ADMM). The architecture of the MG agent are designed, which processes the three control layers via information exchange among neighbouring agents. The proposed framework is validated in a hardware-in-the-loop test-bed, where NMG system with six buses and three MGs is emulated in the OPAL-RT, while the control algorithms are realized by a multi-agent system. The experimental results verify the effectiveness and advantages of the proposed framework, which demonstrates a practical control framework design for NMG systems.
\end{abstract}

Index Terms-Networked microgrids, peer-to-peer architecture, alternating direction method of multipliers, hardware-inthe-loop, multi-agent system.

\section{NOMENCLATURE}

\section{Parameters}

$a_{i j}^{k}$

Communication coefficient between DG $i$ and $j$ in $\mathrm{MG} k$

$a_{k h}$ Communication coefficient between MG $k$ and $h$

$g_{i}^{k}$ Pinning gain of DG $i$ in MG $k$

$g_{k}$

$K_{D G_{i}}^{P}, K_{D G_{i}}^{Q}$ Pinning gain of MG $k$

$K_{k}^{P}, K_{k}^{Q}$ DG $i$

$P_{k}^{\max }, P_{k}^{\min }$ Maximum and minimum real power limitation of MG $k$

The work in this paper was supported in part by Ministry of Education (MOE), Republic of Singapore, under grant AcRF TIER 1 2019-T1-001-069 (RG75/19). Y. Xu's work is supported in part by Nanyang Assistant Professorship from Nanyang Technological University, Singapore, and in part by Singtel Cognitive and Artificial Intelligence Lab for Enterprises (SCALE@NTU), which is a collaboration between Singapore Telecommunications Limited (Singtel) and Nanyang Technological University (NTU) that is funded by the Singapore Government through the Industry Alignment Fund - Industry Collaboration Projects Grant.

Y. Wang and Y. Xu are with Nanyang Technological University, Singapore 639798 (e-mail:wangyulz@hotmail.com).

T.L. Nguyen, R. Caire are with University Grenoble Alpes, G2Elab, F38000 Grenoble, France (tung-lam.nguyen@g2elab.grenoble-inp.fr).

Q.T. Tran is with Alternative Energies and Atomic Energy Commission (CEA), National Institute for Solar Energy (INES), Le Bourget-du-lac, France.

$\begin{array}{ll}Q_{k}^{\max }, Q_{k}^{\min } & \begin{array}{l}\text { Maximum and minimum reactive power limi- } \\ \text { tation of MG } k\end{array} \\ \mathbf{Y} & \text { Admittance matrix of the NMG } \\ \text { Sets } & \text { Set of local constraint of bus } k \\ \mathcal{C}_{k} & \text { Set of bus with MG connection in the NMG } \\ \mathcal{M} & \text { Set of bus in the NMG } \\ \mathcal{N} & \text { Lagrangian multiplier of subsystem at bus } k \\ \text { Variables } & \text { Vector of local voltage variables at bus } k \\ \boldsymbol{\lambda}_{k} & \text { Nominal set points of frequency and voltage } \\ \hat{\mathbf{v}}_{k} & \text { magnitude } \\ \omega^{*}, V^{*} & \text { Nominal frequency and voltage of MG } k \\ \omega_{D G_{k}}^{*}, V_{D G_{k}}^{*} & \text { Secondary control inputs of DG } i \\ \Omega_{D G_{i}}, e_{D G_{i}} & \begin{array}{l}\text { Frequency and voltage of DG } i \\ \omega_{D G_{i}}, V_{D G_{i}}\end{array} \\ \Omega_{k}, e_{k} & \text { Secondary control inputs of MG } k \\ \omega_{M G_{k}}, V_{M G_{k}} & \begin{array}{l}\text { Frequency and voltage magnitude of MG } k \\ \tilde{\mathbf{v}}_{k}\end{array} \\ p_{k}^{L}, q_{k}^{L} & \text { Global variables of subsystem at bus } k \\ P_{k}^{T e r}, Q_{k}^{T e r} & \text { Load demand at bus } k \\ P_{D G_{i}}, Q_{D G_{i}} & \text { Tertiary real and reactive dispatch of MG } k \\ P_{k}, Q_{k} & \text { Real and reactive power of DG } i \\ & k \text { and NMG }\end{array}$

\section{INTRODUCTION}

$\mathbf{M}$ ICROGRIDS (MGs), as fundamental subsystems in smart grids, are integrated with distributed generators (DGs), controllable and non-controllable loads, energy storage systems (ESSs), as well as sophisticated control and communication systems [1], [2]. Typically, the control systems of MGs are developed in a hierarchical way [3]. The hierarchies of MG control are divided into three layers, namely primary, secondary, and tertiary control. The primary control refers to the droop control and the inner control loops of DGs, which is aimed for power sharing with only local measurement [4]. The secondary control functions to restore voltage/frequency deviations caused by droop control, and maintain load sharing according to the rated capacity of DGs [5]. The tertiary control realizes the global optimal and economic operation of MGs [6].

A single MG system may not ensure a reliable operation in some extreme circumstances such as main grid failures and nature disasters. To enhance entire system's reliability and resilience, one of the feasible solutions is to interconnected MGs in a certain area to form the networked microgrid (NMG) system [7], [8]. The practical scenarios of NMG systems include active distribution networks with residential microgrids [9], building microgrids community [10], maritime 
electrical systems with seaport and shipboard microgrids [11], etc. The NMG systems can be further categorized depending on their electrical structures, such as voltage levels, type of current, way of connections, etc. For example, according to the boundaries and distances among MGs, low-voltage (LV) MGs can be connected by either LV tie-line or boosting transformers into a MV network [12]. The type of current in the NMGs can be either AC, DC or hybrid AC/DC [13]. The MGs can be interconnected either in serial or in parallel [14]. In this paper, the scenario that AC NMG system with multiple LV MGs integrated into a MV network in parallel is under consideration.

Compared with the control and operation of the single MG system, the methodologies for NMG system can be more challenging since more electrical components and communication infrastructures are coupled together. To ensure the stable and flexible operation of NMGs, several studies endeavor to apply the existing single MG control framework into NMG systems. [12], [15]-[17]. In [12], a two layer distributed hierarchical control scheme is proposed for AC NMG systems in power distribution networks. In [15], the hierarchical distributed control framework for DC microgrids are extended to MG clusters. In [16], the droop-based control and small signal stability of PV-based multiple MG clusters are investigated. In [17], a hierarchical consensus control framework has been proposed for managing multiple MG clusters in Energy Internet with a multi-site experimental validation. However, the coordination with upper-level optimized dispatch is not studied in the above research. In addition, due to ownership and privacy concerns, the data and communication access of each $\mathrm{MG}$ is usually limited. It makes a distributed control structure with minor data exchange with MG level control highly preferred.

On the other hand, many research works have been conducted to realize optimal and economical operation in NMGs [18]-[23]. The energy management system plays the vital role in operation of NMGs, which has attracted much research interest. The existing research investigates energy management strategies for NMGs with various features, such as the resilient and privacy [18], multi-agent energy management [19], cooperative energy and reserve scheduling [20], stochastic predictive control [21], hierarchical management [22], and restoration and reconfiguration after disasters [23]. In these works, the coordination with lower-level control and realization is not considered by these research. The multilayer control and optimization methods for NMG systems are separately discussed and a lack of the coordination of layers. This motivates us to investigate this problem, and propose a peer-to-peer (P2P) control architecture for NMG systems with fully distributed and hierarchical framework.

Recently, some research efforts have been made on control and optimization of the 'system of system' problem in NMGs [24]-[26]. By simplifying the inner dynamics of a single MG as a second-order generator model, the authors in [24] provide a distributed optimal tie-line power flow control strategies for interconnected AC MGs. In [25], a distributed hierarchical control is proposed for DC MG clusters, but the method still deals with the coordination of each DG unit other than the MG system. A distributed optimal control framework is proposed for multiple MGs in distribution networks, which contains the optimization of the NMG and the control within each MG [26]. A comparison of control architecture and validation methodologies is further made in Table I. We notice several major limitations of these existing studies: (i) The fully distributed control structure with inter layer coordination in NMGs are not well addressed. (ii) The tertiary control is modelled as economic dispatch problem in [24] and [25], which ignores the network constraints in the NMG level system. (iii) The multi-layer, agent-based control framework for multiple MGs, which supports P2P operation of NMG system has not been systematically introduced. (iv) Last but not least, the hardware realization of $\mathrm{P} 2 \mathrm{P}$ control framework in multi-agent systems (MASs) for NMGs, as an emerging and practical topic, has rarely been investigated.

In order to narrow the gap between theoretical research and practical deployments, this paper presents a $\mathrm{P} 2 \mathrm{P}$ control architecture for NMGs and its implementation on a hardware MAS. The control system is constructed to achieve multiple functionalities in different layers and multiple time scales. The primary control functions to bridge MG level control of DGs and NMG level control of MGs. The secondary control is to maintain frequency/voltage at reference values and achieve arbitrary power-sharing. The tertiary control will minimize the network power loss in the NMG system. These three control layers are integrated into a $\mathrm{MG}$ agent, where signal exchange among layers and agents is specifically designed. In each MG agent, three processes run in parallel corresponding to three control layers: the primary process executes the MG droop control, the secondary process implements the distributed consensus control, and the tertiary process operates the alternating direction method of multipliers (ADMM) algorithm. Furthermore, the designed agents are realized on a hardware MAS platform, where agents are operated in a realistic communication network as asynchronous devices. The hardware-in-the-loop (HiL) test-bed, which consists of a realtime simulator and a MAS, is used to verify the performance of the proposed control and design framework. Compared with previous research, the major contributions of this papers are:

- A P2P control architecture considering multi-layers and multi-agent interaction is proposed for the NMG systems.

- The agent design on embedded system is systematically introduced with detailed information exchange and timescale coordination.

- The proposed P2P architecture is validated on a MAS HiL platform to test its the effectiveness and practicability.

The paper is structured as follows. Section II provides preliminaries of this paper. Section III introduces the proposed P2P control architecture for NMGs. Then, the design and implementation of each MG agent are presented in Section IV. Section V presents the case studies of NMG in the HiL testbed. Finally, in Section VI, conclusions are drawn.

\section{PREliminaries}

\section{A. Cyber-Physical Structure of NMG System}

A NMG system is a complex interacted cyber-physical system, which consists of highly coupled electrical and com- 
TABLE I: Comparison of control architecture and validation methodologies

\begin{tabular}{|c|c|c|c|c|c|c|c|}
\hline \multirow{2}{*}{ Reference } & \multicolumn{5}{|c|}{ Architecture } & \multicolumn{2}{|c|}{ Validation } \\
\hline & Primary & Secondary & Tertiary & Distributed & Centralized & Simulation & HiL \\
\hline [12] & $\sqrt{ }$ & $\checkmark$ & $x$ & $\sqrt{ }$ & $x$ & $\sqrt{ }$ & $x$ \\
\hline [15], [17] & $\sqrt{ }$ & $\checkmark$ & $x$ & $\sqrt{ }$ & $x$ & $x$ & $\sqrt{ }$ \\
\hline [16] & $\sqrt{ }$ & $\sqrt{ }$ & $x$ & $x$ & $\sqrt{ }$ & $\sqrt{ }$ & $x$ \\
\hline$[18],[20]-[23]$ & $x$ & $x$ & $\sqrt{ }$ & $x$ & $\sqrt{ }$ & $\sqrt{ }$ & $x$ \\
\hline [19] & $x$ & $x$ & $\sqrt{ }$ & $\sqrt{ }$ & $x$ & $\sqrt{ }$ & $x$ \\
\hline$[24],[26]$ & $\sqrt{ }$ & $x$ & $\sqrt{ }$ & $\sqrt{ }$ & $x$ & $\checkmark$ & $x$ \\
\hline [25] & $\sqrt{ }$ & $\sqrt{ }$ & $\sqrt{ }$ & $\sqrt{ }$ & $x$ & $\sqrt{ }$ & $x$ \\
\hline Proposed & $\checkmark$ & $\sqrt{ }$ & $\checkmark$ & $\checkmark$ & $x$ & $x$ & $\checkmark$ \\
\hline
\end{tabular}

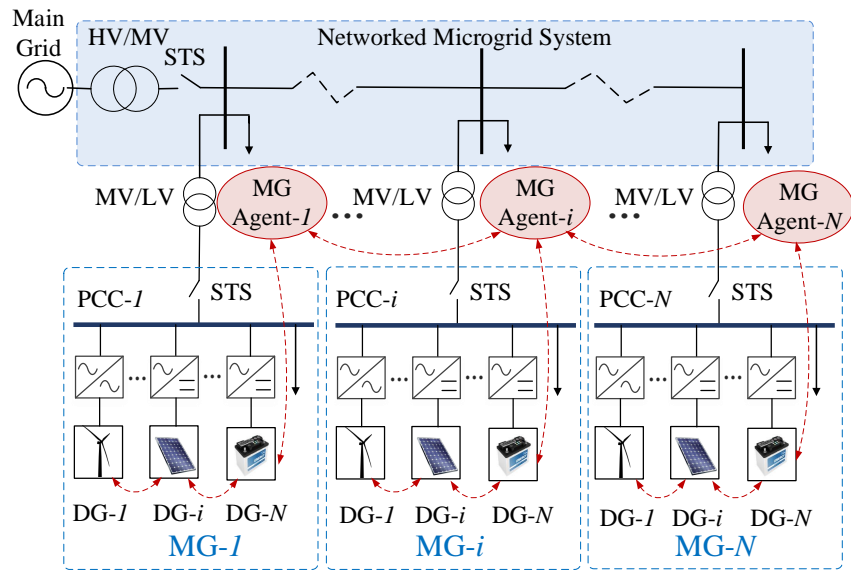

Fig. 1: Typical schematic of the networked microgrid systems

munication systems [27]. A single-line exemplary electrical structure of NMG system is demonstrated in Fig. 1. Normally, a NMG system can be operated in either islanded or gridconnected modes from the main grid. In this paper, an islanded NMG system are investigated where the static transfer switch (STS) isolate the NMG with the main grid. Besides, Each MG is integrated with a group of dispatch-able DGs and local loads, which are connected to a point of common coupling (PCC). Then, each MG is connected to a certain bus of MV network in the NMG system via LV/MV transformers. The renewable energy resources (RESs) working at maximum power point tracking mode and constant power loads (CPLs) at the same bus can be viewed as the lumped load [17]. Due to the focus of this paper is the AC NMG, the DG units are considered as power converters with DC voltage sources. The communication system in an NMG system contains the upper-level communication network among MGs and the lower communication network among DGs within each MG, as shown in Fig.1. In the communication network of NMG system, each MG agent measures the tie-line information of each MG and exchange with neighbouring agents, where the reference frequency and voltage signals are generated. Then the reference signals are tracked by all the DGs based on existing distributed secondary control for single MGs. In next subsection, the typical distributed secondary control scheme of a single $\mathrm{MG}$, as the preliminary knowledge of NMG level design, is introduced.

\section{B. Distributed Control for a Single $M G$}

Droop control is widely used to control the magnitude of voltage and frequency in case of inverter interfaced DGs in islanded MGs. The droop characteristic for DG $i$ is shown as follows [3], [4]:

$$
\begin{aligned}
\omega_{D G_{i}} & =\omega_{M G_{k}}^{*}-K_{D G_{i}}^{P} P_{D G_{i}} \\
V_{D G_{i}} & =V_{M G_{k}}^{*}-K_{D G_{i}}^{Q} Q_{D G_{i}}
\end{aligned}
$$

where $\omega_{M G_{k}}^{*}$ and $V_{M G_{k}}^{*}$ are the nominal frequency and voltage amplitude of the MG $k . K_{D G_{i}}^{P}$ and $K_{D G_{i}}^{Q}$ are droop coefficients, which are commonly chosen based on the output power rating.

The secondary control is to track the reference values fed by NMG level control, and achieve the DG's accurate power sharing within each MG. Distributed secondary control including linear and non-linear methods have been widely investigated by state-of-the-art. Based on the proposed methods in [5], [27], [28], for $i$ th DG, a linear control for frequency restoration and real power sharing is designed as follow:

$$
\begin{aligned}
\omega_{D G_{i}} & =\omega_{M G_{k}}^{*}-K_{D G_{i}}^{P} P_{D G_{i}}+\Omega_{D G_{i}} \\
\dot{\Omega}_{D G_{i}} & =\sum_{j=1}^{N_{M G_{k}}} a_{i j}^{k}\left(\omega_{D G_{j}}-\omega_{D G_{i}}\right)+g_{i}^{k}\left(\omega_{M G_{k}}-\omega_{D G_{i}}\right) \\
& +\sum_{j=1}^{N_{M G_{k}}} a_{i j}^{k}\left(K_{D G_{j}}^{P} P_{D G_{j}}-K_{D G_{i}}^{P} P_{D G_{i}}\right)
\end{aligned}
$$

Similarly, a linear control for voltage regulation and reactive power sharing is designed as follow:

$$
\begin{aligned}
V_{D G_{i}} & =V_{M G_{k}}^{*}-K_{D G_{i}}^{Q} Q_{D G_{i}}+e_{D G_{i}} \\
\dot{e}_{D G_{i}} & =\sum_{j=1}^{N_{M G_{k}}} a_{i j}^{k}\left(e_{D G_{j}}-e_{D G_{i}}\right)+g_{i}^{k}\left(V_{M G_{k}}-V_{D G_{i}}\right) \\
& +\sum_{j=1}^{N_{M G_{k}}} a_{i j}^{k}\left(K_{D G_{j}}^{Q} Q_{D G_{j}}-K_{D G_{i}}^{Q} Q_{D G_{i}}\right)
\end{aligned}
$$

where $\Omega_{D G_{i}}$ and $e_{D G_{i}}$ are control signals from secondary control. $a_{i j}^{k}$ is the communication coefficient between DGs $i$ and $j$ in MG $k, a_{i j}^{k}>0$ if there is a link, otherwise, $a_{i j}^{k}=0$. $g_{i}^{k}$ is the pinning gain of the DG $i$ in $k$ th MG, where $g_{i}^{k}>0$ if the DG can directly receive $\omega_{M G_{k}}$ and $V_{M G_{k}}$, and $g_{i}^{k}=0$ 
otherwise. $N_{M G_{k}}$ is the total number of DGs in MG $k . \omega_{M G_{k}}$ and $V_{M G_{k}}$ are the frequency and voltage reference values calculated by NMG level control.

\section{Proposed PeEr-to-Peer Control Architecture}

In the NMG control hierarchies, the key points differentiate each control level are the response speed and infrastructure requirement (e.g. communication requirement). When a small disturbance occurs, primary control reacts immediately to provide fast response for frequency or voltage variations in the MG. Then the secondary control activate for frequency/voltage regulation and power sharing. The tertiary control, as a highest and slowest response control level, will operate to minimize the network loss. The entire P2P control framework of NMG systems can be realized based on neighbouring communications. The three control layers with a fully distributed structure are introduced in following subsections.

\section{A. Primary Control Layer in NMG}

Similar to the droop control of DGs in a single MG, a droop control is proposed for each MG to operate autonomous with only local measurement. The NMG includes $N$ buses with the set of buses $\mathcal{N}$, the set of MG $\mathcal{M}$, and the set of lines $\mathcal{V}$. The droop control for MG $k$ is defined as follows:

$$
\begin{aligned}
\omega_{M G_{k}} & =\omega^{*}-K_{k}^{P} P_{k}, k \in \mathcal{M} \\
V_{M G_{k}} & =V^{*}-K_{k}^{Q} Q_{k}, k \in \mathcal{M}
\end{aligned}
$$

where $\omega^{*}$ and $V^{*}$ are the nominal frequency and voltage amplitude at NMG level. $P_{k}$ and $Q_{k}$ are the power exchange among MG $k$ and the NMG. $K_{k}^{P}$ and $K_{k}^{Q}$ are droop coefficients of each MG.

Remark 1: Similar to the droop coefficients selection for DG units, the real and reactive droop coefficients of a MG can be selected based on its allowable operation range [3], [12]. In this way, the $K_{k}^{P}$ and $K_{k}^{Q}$ are chosen as:

$$
\begin{aligned}
K_{k}^{P} & =\frac{\omega^{\max }-\omega^{\text {min }}}{P_{k}^{\text {max }}-P_{k}^{\text {min }}} \\
K_{k}^{Q} & =\frac{V^{\text {max }}-V^{\text {min }}}{Q_{k}^{\text {max }}-Q_{k}^{\text {min }}}
\end{aligned}
$$

where $\omega^{\max }$ and $\omega^{\text {min }}$ are the allowable maximum and minimum frequency, respectively. $V^{\max }$ and $V^{\min }$ are the allowable maximum and minimum voltage, respectively. $P_{k}^{\max }$ and $P_{k}^{\text {min }}$ are the maximum and minimum power outputs of MG $k \cdot Q_{k}^{\max }$ and $Q_{k}^{\min }$ are the maximum and minimum power outputs of MG $k$. It is noted that the droop coefficients of MGs can be also designed for other objectives.

\section{B. Secondary Control Layer in NMG}

The distributed secondary control of NMGs is to achieve three objectives: 1) frequency restoration, 2) point of common coupling (PCC) bus voltage restoration, and 3) arbitrary power sharing among MGs.

Based on consensus algorithms, the distributed secondary control laws for each MG can be designed as follows:

$$
\begin{aligned}
\omega_{M G_{k}} & =\omega^{*}-K_{k}^{P} P_{k}+\Omega_{M G_{k}} \\
\dot{\Omega}_{M G_{k}}= & \sum_{h=1}^{M} a_{k h}\left(\omega_{h}-\omega_{k}\right)+g_{k}\left(\omega^{*}-\omega_{k}\right) \\
& +\sum_{h=1}^{M} a_{k h}\left[K_{h}^{P}\left(P_{h}-P_{h}^{T e r}\right)-K_{k}^{P}\left(P_{k}-P_{k}^{T e r}\right)\right] \\
V_{M G_{k}} & =V^{*}-K_{k}^{Q} Q_{k}+e_{M G_{k}} \\
\dot{e}_{M G_{k}}= & \sum_{h=1}^{M} a_{k h}\left(e_{h}-e_{k}\right)+\left(V^{*}-V^{P C C}\right) \\
& +\sum_{h=1}^{M} a_{k h}\left(K_{h}^{Q}\left(Q_{h}-Q_{h}^{T e r}\right)-K_{k}^{Q}\left(Q_{k}-Q_{k}^{T e r}\right)\right) \\
& k, h \in \mathcal{M}
\end{aligned}
$$

where $\Omega_{M G_{k}}$ and $e_{M G_{k}}$ are control signals from secondary control of the NMG system. $a_{k h}$ is the communication coefficient between MGs $k$ and $h . g_{k}$ is the pinning gain of the MG $k$. $M$ is the total number of MGs in the NMG.

The NMG system with proposed control laws in (9) and (10) will converge to:

$$
\begin{gathered}
\lim _{t \rightarrow \infty}\left|\omega^{*}-\omega_{k}(t)\right|=0 \\
\lim _{t \rightarrow \infty}\left|V^{*}-V^{P C C}(t)\right|=0 \\
\lim _{t \rightarrow \infty}\left|K_{h}^{P}\left(P_{h}(t)-P_{h}^{T e r}\right)-K_{k}^{P}\left(P_{k}(t)-P_{k}^{T e r}\right)\right|=0 \\
\lim _{t \rightarrow \infty}\left|K_{h}^{Q}\left(Q_{h}(t)-Q_{h}^{T e r}\right)-K_{k}^{Q}\left(Q_{k}(t)-Q_{k}^{T e r}\right)\right|=0
\end{gathered}
$$

Remark 2: According to the proposed distributed secondary control, the system frequency of each bus in NMG system will be restored to the reference value. The point of common coupling voltage of the NMG system will also be restored to its reference value. The real and reactive power will follow the dispatch signal $P_{k}^{T e r}, Q_{k}^{T e r}$ from tertiary control, while the mismatch due to load variations will be shared according to droop coefficients $K_{k}^{P}$ and $K_{k}^{Q}$ defined in (5) and (6) for each MG.

\section{Tertiary Control Layer in NMG}

The tertiary control is aimed to provide an optimized operation of the NMG system in a distributed manner. In this paper, the control objective is to minimize the total network power losses in the MV network of the NMG. Since the dynamics of frequency and voltage restoration are much faster, the values of frequency and voltage can be considered in steady-state by the tertiary optimization. The tertiary control solves the optimal power flow (OPF) problem and returns the real and reactive power dispatch orders to the secondary control layers (i.e. $P_{k}^{T e r}, Q_{k}^{T e r}$ in (9) and (10)). The proposed control framework will follow the dispatch orders to make the system operate around the optimal state. In this paper, the ADMM algorithm is applied to solve the OPF problem with only neighboring data exchange.

\section{1) Alternating Direction Method of Multipliers}

Alternating direction method of multipliers algorithm is systematically introduced for solving a distributed optimization 
problem in [29]. The main advantage of using ADMM is that it inherits the benefits of dual decomposition and augmented Lagrangian methods for constrained optimizations. We now consider the problem in a general decomposition structure with the objective and constraint terms split into $K$ parts:

$$
\begin{array}{ll}
\text { minimize } & \sum_{k=1}^{K} f_{k}\left(\mathbf{x}_{k}\right) \\
\text { subject to } & \mathbf{x}_{k} \in \mathcal{C}_{k}, \quad k=1, \ldots, K \\
& \mathbf{x}_{k}-\tilde{\mathbf{z}}_{k}=0, \quad k=1,2, \ldots, K
\end{array}
$$

where $\mathbf{x}_{k}$ is local variable, $\mathbf{z}$ is global variable, $\tilde{\mathbf{z}}_{k}$ is the fraction of the variable $\mathbf{z}$ that local variable $\mathbf{x}_{k}$ should be, $\mathcal{C}_{k}$ is the local constraint.

The augmented Lagrangian of the problem (15) related to the coupling constraint is given by:

$L_{\rho}\left(\mathbf{x}_{k}, \tilde{\mathbf{z}}_{k}, \boldsymbol{\lambda}_{k}\right)=\sum_{k=1}^{K}\left(f_{k}\left(\mathbf{x}_{k}\right)+\boldsymbol{\lambda}_{k}^{T}\left(\mathbf{x}_{k}-\tilde{\mathbf{z}}_{k}\right)+(\rho / 2)\left\|\mathbf{x}_{k}-\tilde{\mathbf{z}}_{k}\right\|_{2}^{2}\right)$

where $\boldsymbol{\lambda}_{k}$ is the dual variables associated with the equality constraint, $\rho \in \mathbb{R}$ is the Lagrangian step parameter.

\section{2) Network Loss Minimization}

The OPF problem with the objective of minimizing total real power losses is formulated as:

$$
\underset{\hat{\mathbf{v}}}{\operatorname{minimize}} \hat{\mathbf{v}}^{T} \cdot \mathbf{z}^{p} \cdot \hat{\mathbf{v}}
$$

subject to

at $k=1, \cdots, N$

$$
\begin{aligned}
& P_{k}^{\text {min }} \leq \hat{\mathbf{v}}_{k}^{T} \cdot \mathbf{z}_{k}^{p} \cdot \hat{\mathbf{v}}_{k}+p_{k}^{L} \leq P_{k}^{\max }, k \in \mathcal{M} \\
& Q_{k}^{\text {min }} \leq \hat{\mathbf{v}}_{k}^{T} \cdot \mathbf{z}_{k}^{q} \cdot \hat{\mathbf{v}}_{k}+q_{k}^{L} \leq Q_{k}^{\max }, k \in \mathcal{M} \\
& \hat{\mathbf{v}}_{k}^{T} \cdot \mathbf{z}_{k}^{p} \cdot \hat{\mathbf{v}}_{k}+p_{k}^{L}=0, k \notin \mathcal{M} \\
& \hat{\mathbf{v}}_{k}^{T} \cdot \mathbf{z}_{k}^{q} \cdot \hat{\mathbf{v}}_{k}+q_{k}^{L}=0, k \notin \mathcal{M} \\
&\left(\mathbf{v}_{k}^{\text {min }}\right)^{2} \leq\left(\mathbf{v}_{k}^{r e}\right)^{2}+\left(\mathbf{v}_{k}^{i m}\right)^{2} \leq\left(\mathbf{v}_{k}^{\max }\right)^{2}
\end{aligned}
$$

where $\hat{\mathbf{v}}$ is indicated by $\hat{\mathbf{v}}=\left[\begin{array}{c}\mathbf{v}^{r e} \\ \mathbf{v}^{i m}\end{array}\right] \in \mathbb{R}^{2 N}, \hat{\mathbf{v}}=\mathbf{v}^{r e}+\mathbf{v}^{i m}$ is the nodal voltage vector; $\hat{\mathbf{v}}_{k} \in \mathbb{R}^{2 N_{k}}$ is the vector implied from $\hat{\mathbf{v}}$ by eliminating elements not involved in bus $k ; N_{k}$ is the number of buses including bus $k$ and buses having connection with bus $k$; $P_{k}^{\min }, P_{k}^{\max }, Q_{k}^{\min }$ and $Q_{k}^{\max }$ are real and reactive power limitation of $\mathrm{MG}$ at bus $k ; p_{k}^{L}$ and $q_{k}^{L}$ are load power at bus $k ; \mathbf{v}_{k}^{\min }$ and $\mathbf{v}_{k}^{\max }$ are bus voltage thresholds. The matrices $\mathbf{z}^{p}$ and $\mathbf{z}^{q} \in \mathbb{R}^{2 N \times 2 N}$ are indicated by:

$$
\mathbf{z}^{p}=\left[\begin{array}{cc}
\mathbf{G} & -\mathbf{B} \\
\mathbf{B} & \mathbf{G}
\end{array}\right] \quad \mathbf{z}^{q}=\left[\begin{array}{cc}
-\mathbf{B} & \mathbf{- G} \\
\mathbf{G} & \mathbf{- B}
\end{array}\right]
$$

where $\mathbf{Y}=\mathbf{G}+j \mathbf{B}$ is the admittance matrix of the grid system, which is the inverse of the impedance matrix. $\mathbf{z}_{k}^{p}$ and $\mathbf{z}_{k}^{q} \in \mathbb{R}^{2 N_{k} \times 2 N_{k}}$ are the matrices implied from $\mathbf{z}^{p}$ and $\mathbf{z}^{q}$ respectively, obtained by writing constraints in the quadratic form.

In order to solve the OPF problem in a distributed way, we need to split the problem (17)-(22) into subsystems. The number of the subsystems corresponds to the number of buses in the NMG. The total real power losses can be expressed by decomposing the function into $N$ parts:

$$
\hat{\mathbf{v}}^{T} \cdot \mathbf{z}^{p} \cdot \hat{\mathbf{v}}=\sum_{k=1}^{N} \hat{\mathbf{v}}_{k}^{T} \cdot \mathbf{z}_{k}^{p} \cdot \hat{\mathbf{v}}_{k}
$$

The sub-problem at bus $k$ involves in $N_{k}$ buses and can be expressed as follows.

- If $k \notin \mathcal{M}$ :

$$
\begin{array}{ll}
\underset{\hat{\mathbf{v}}_{k}}{\operatorname{minimize}} & \hat{\mathbf{v}}_{k}^{T} \cdot \mathbf{z}_{k}^{p} \cdot \hat{\mathbf{v}}_{k} \\
\text { subject to } & (20),(21),(22)
\end{array}
$$

- If $k \in \mathcal{M}$ :

$$
\begin{array}{ll}
\underset{\hat{\mathbf{v}}_{k}}{\operatorname{minimize}} & \hat{\mathbf{v}}_{k}^{T} \cdot \mathbf{z}^{p} \cdot \hat{\mathbf{v}}_{k} \\
\text { subject to } & (18),(19),(22)
\end{array}
$$

where $\hat{\mathbf{v}}_{k}$ is the local variable.

The OPF problem is therefore formulated in the general consensus problem as presented in (15) and can be solved in a distributed way by ADMM. The coupling constraint is:

$$
\hat{\mathbf{v}}_{k}-\tilde{\mathbf{v}}_{k}=0
$$

where $\tilde{\mathbf{v}}_{k}$ is the global variable representing the collection of the related components of $\hat{\mathbf{v}}$ that map into subsystem $k$.

\section{Multi-Agent System Realization}

The P2P control architecture proposed in previous section will be realized on a MAS with neighboring communications. An agent refers to an entity owning capabilities of receiving local measurement, communicating with other agents, processing calculations, and then returning proper signals to the DG level controllers. In the MAS with $\mathrm{P} 2 \mathrm{P}$ architecture, instead of collecting all data to a central controller, each agent only requires local and adjacent information but can return system level signals to achieve global objectives. The neighbor agents are defined based on the electrical connection of a NMG system.

\section{A. Agent}

Agents are designed to run as independent entities, which relies on limited system knowledge. Intuitively, each agent updates the state of the power network, processes the calculation and then returns control decisions. Depending on functionalities of agents, they are classified into two types, i.e. MG-agents and load-agents. MG-agents are located at MG buses to manage the power exchange between MG and NMG. Load-agents, which locate at load buses, measure local information, and transmit the neighbouring information. Fig. 2 illustrates the multi-layer control scheme realized in the MG-agent. The local controller is the primary control which implements the droop control with only local measurements. According to the proposed fully distributed multi-layer control, we design the agents for a practical implementation of the system. To achieve all control objectives simultaneously, the agent contains three separate processes running in parallel: the primary process, the secondary process and the tertiary process. 


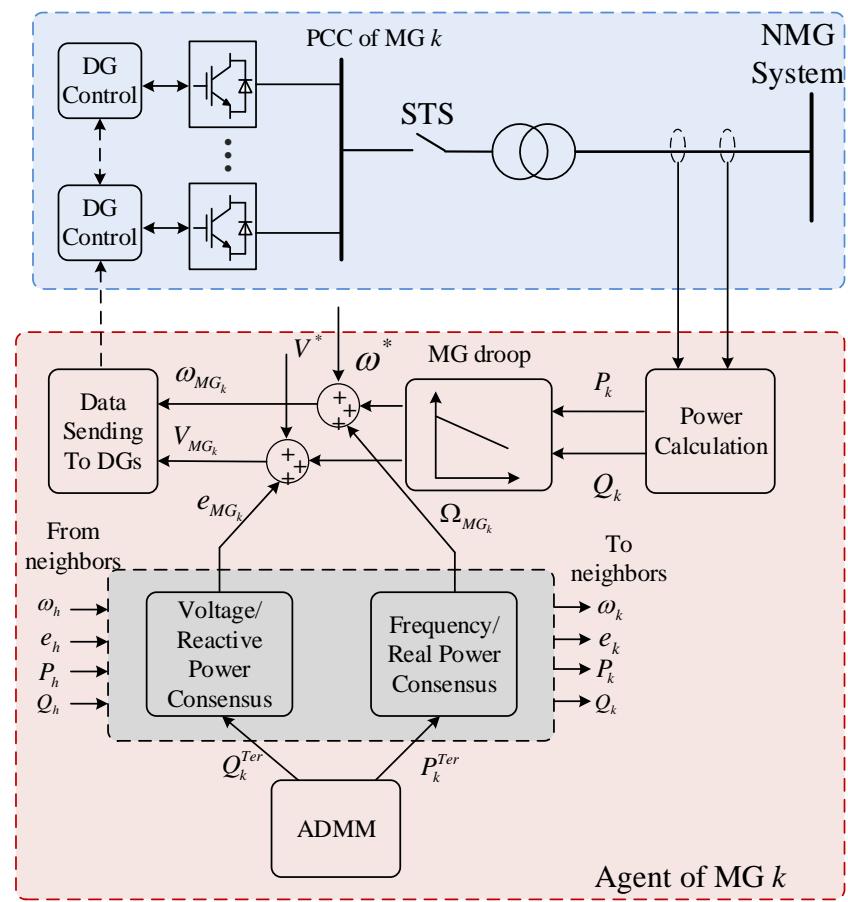

Fig. 2: Multi-layer control algorithms in an agent.

\section{B. Primary Process}

In the primary process, each MG-agent operates its virtual droop control laws in (5) and (6) to calculate tracking reference values $\omega_{M G_{k}}$ and $V_{M G_{k}}$ for DGs within the MG. This control process only require local measurement, which will always be operated to ensure system stable operation in absence of secondary and tertiary control process.

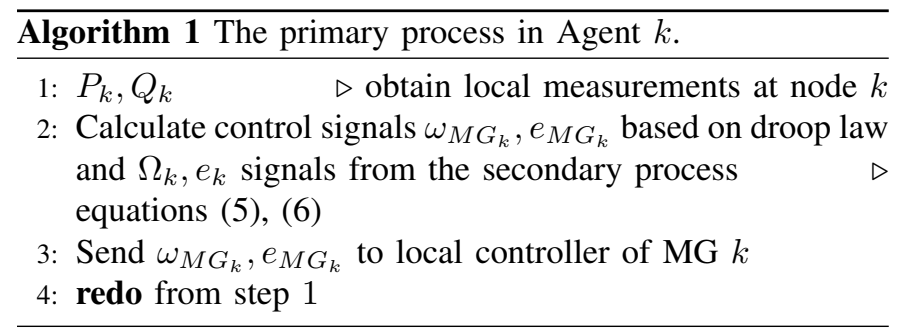

\section{Secondary Process}

All MGs with its DGs contribute to secondary control process to recover system voltage and frequency. In the distributed control scheme, the MG agents iteratively exchange information sensed locally for the computation of the consensus laws (9) and (10). Due to the fact that MG-agents may not connect together directly but through several edges in the NMG graph. Therefore, each agent processes several intermediate iterations, called a consensus loop, for collecting and distributing data before computing the control laws.

Algorithm 2 describes the iterative step for the secondary process in a consensus loop. Initially, agents collect local measurement $\left\{\omega_{k}, e_{k}, P_{k}, Q_{k}\right\}$ from devices and exchange message $\left\{\omega_{k}, e_{k}, K_{k}^{P}\left(P_{k}-P_{k}^{T e r}\right), K_{k}^{Q}\left(Q_{k}-Q_{k}^{T e r}\right)\right\}$ among neighbors. Control signals will be computed and sent to controllers when a loop is finished. The frequency reference value in the local controller is adjusted by the signal $\Omega_{k}$; while the voltage reference value is adjusted by the signal $e_{k}$.
Algorithm 2 The secondary process in Agent $k$.

1: $\mathcal{N}^{k}$

$\triangleright$ list of neighborhood agents

2: $\omega_{k}, e_{k}, P_{k}, Q_{k} \quad \triangleright$ obtain local measurements at node $k$

3: Take several steps of distributing local measurements collecting values from MG neighbors

4: Calculate control signals $\Omega_{M G_{k}}, e_{M G_{k}}$ based on local measurements, and neighborhood information and signals $P_{k}^{T e r}, Q_{k}^{T e r}$ from the tertiary process $\triangleright$ equations (9), (10)

5: Send $\Omega_{k}, e_{k}$ to the primary process

6: redo from step $1 \quad \triangleright$ start a new consensus loop

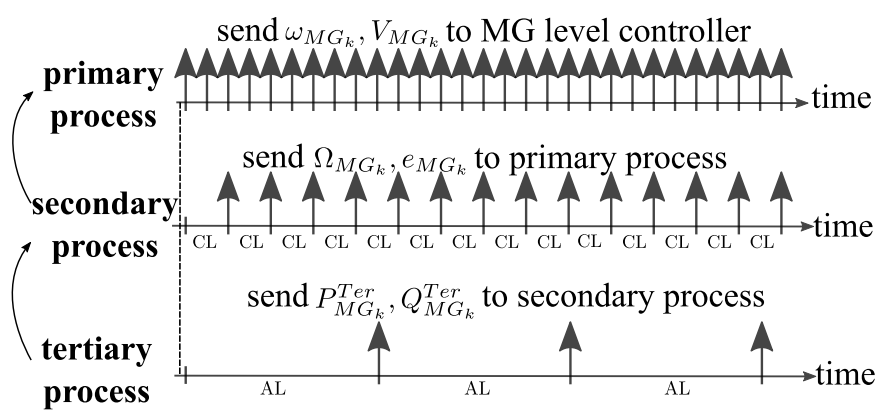

CL: consensus loop AL: ADMM loop

Fig. 3: Three parallel processes in MG-agent $k$.

\section{Tertiary Process}

This process runs the ADMM algorithm. The measurement inputs are $P_{k}, Q_{k}$ and the messages exchanged with neighbors within an iteration are transferred via the same channels used by the secondary process. The implementation of this process is presented in Algorithm 3. Considering MG-agents, the optimal result when finishing an ADMM loop, which is the reference real and reactive power, will be sent to the secondary process to update $P_{k}^{T e r}$ and $Q_{k}^{T e r}$ in the control law as shown in (9) and (10).

We call a ADMM loop is the duration from the moment the agent receives measurements from the device to the moment the agent sends the control signals to the local controller. Agents execute the loops consecutively to always seek the optimal points for DG outputs. The loop begins from iteration 1 and finished at iteration $I_{0}^{a d m m}$ when reaching the consensus of local variables and global variables. When triggering, the agents do not know about system states. Therefore, at the first loop, we can refer to the cold start case. In this case, the initial guess of the global variables in each agent is set as $\mathbf{v}_{k}^{r e}(0)=\mathbf{v}^{\max }, \mathbf{v}_{k}^{i m}(0)=\mathbf{0}$, the initial guess of the Lagrangian multipliers is set to zeros $\boldsymbol{\lambda}_{0}^{k}=\mathbf{0}$. Then, from the second ADMM loop, the warm start can be applied to enhance the convergence. In this case, the starting points of the global variables and the Lagrangian multipliers are the solutions of the previous ADMM loop. In other words, the starting point is the current state of the system.

Remark 3: Fig. 3 clarifies the operation of the processes in a MG-agent. Intuitively, in the MG-agents, the primary process updates $\omega_{M G_{k}}$ and $V_{M G_{k}}$ for DGs in MG level controllers. The secondary process sends control signals $\Omega_{M G_{k}}$ and $e_{M G_{k}}$ to the local primary process to remain frequency and voltage 


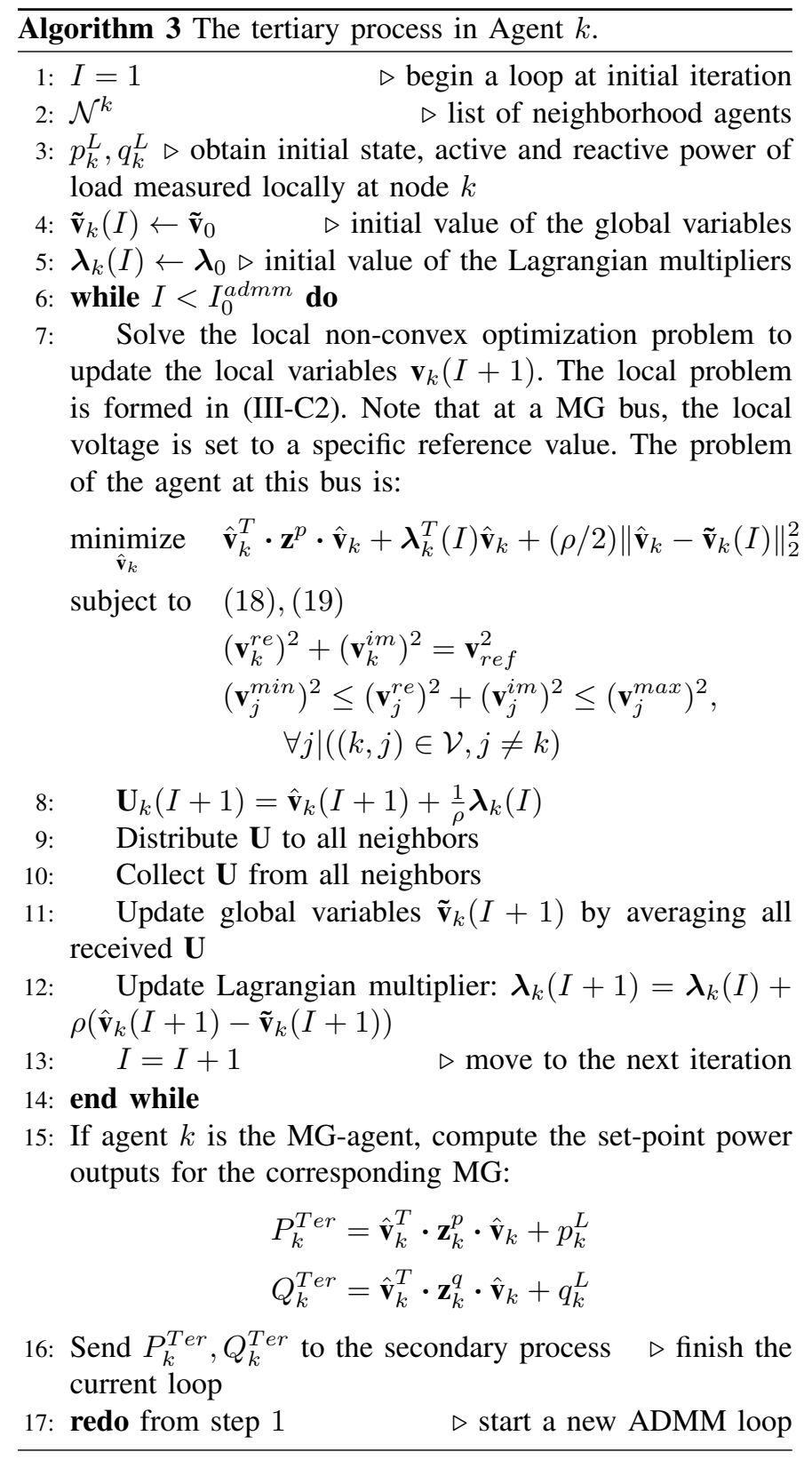

at references as well as to adjust the power output of MG. The power dispatch order are kept at constant values until the tertiary process finishes a ADMM loop and update new $P_{k}^{\text {Ter }}$ and $Q_{k}^{T e r}$. The three processes run in different time scales. The lower process holds signal values from the upper process until receiving new samples when the upper process finishes a loop.

\section{THE HARDWARE-IN-THE-LOOP EXPERIMENTAL RESULTS}

\section{A. Experimental Setup and NMG Test-bed}

This section presents the validation of the agent design with the proposed control framework. We consider a NMG system with six buses operated in the islanded mode. The NMG test system contains three parallel MGs, where MG-1, MG-2, and MG-6 have 4, 3, 3 DGs, respectively. Loads are located at the remain buses. colorred Fig. 5 demonstrate the physical and cyber topology of the test NMG system, where

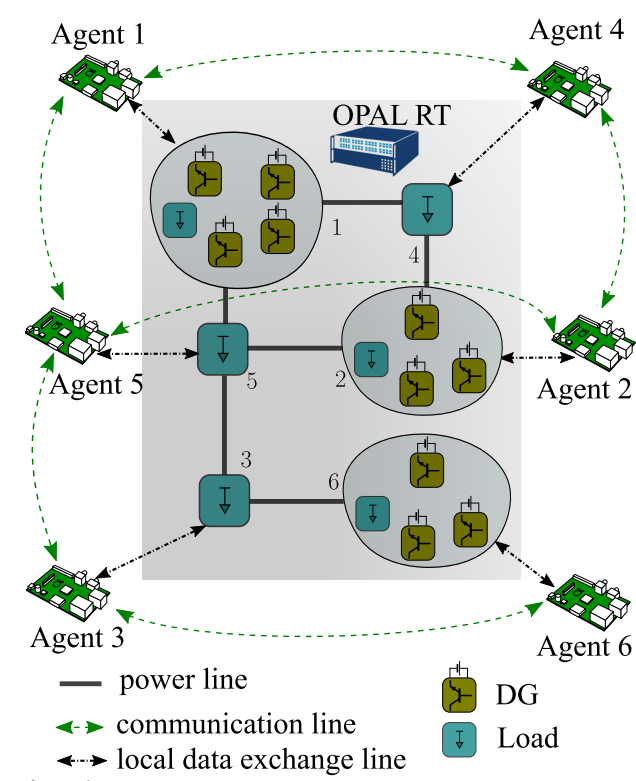

Fig. 4: The test case NMG in the layer structure.

the communication topology among agent is designed to be the same as he electrical network in NMG level. The parameters of the test NMG system are shown in Table II. The experimental setup in the laboratory includes two main parts, which are further illustrated as follows.

\section{1) Physical System}

The physical system covers all the electrical components in the NMG and local controllers of DGs in MGs. It is simulated to run in a real-time simulator OPAL-RT. All the measurement of NMGs required by each control layer will be sent to each agent. The agent will return the calculated control signal $\omega_{M G_{k}}$ and $V_{M G_{k}}$ to the MG level control in OPAL-RT. The signal exchange between agent and OPAL-RT is through user datagram protocol (UDP).

2) Cyber System

The cyber system constitutes the MAS with six Raspberry Pis and a local communication network. The Raspberry Pi 3 Model $\mathrm{B}+$ is used as the agent which operates the proposed control algorithms independently. The data exchange among agents are realised by cable communications though a network switch. The communication among agents is realized by TCP/IP protocol, while the communication between agent and OPAL-RT is through user datagram protocol (UDP). An agent hosted in a Raspberry $\mathrm{Pi}$ is a program written in python language. The MAS is realized by using Google Remote Procedure Call (gRPC) framework. gRPC uses protocol buffers, which has a slightly simplified syntax for serializing structured data, for transferring messages. The communication among agents is achieved in a client/server manner using gRPC and can be configured to any network topology. In gRPC based communication process, each agent is a server that waits for incoming messages and also can dispatch messages to corresponding method calls due to the fact that it is also a client of neighbor servers. Fig. 5 shows the structure of the agent design based on gRPC.

\section{B. Experimental Results}

The HiL experiment test last $480 \mathrm{~s}$ is conducted to validate the performance of the proposed method. The collecting 


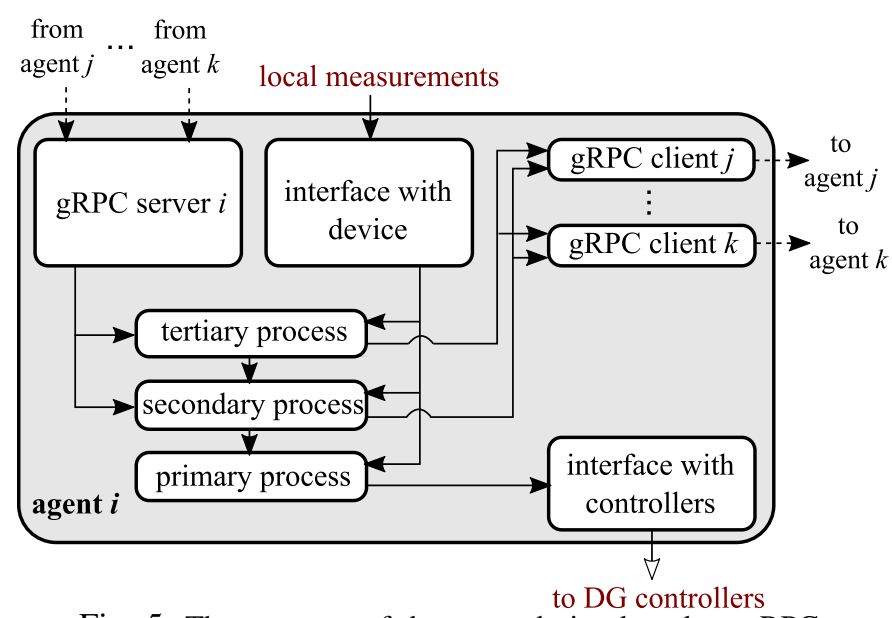

Fig. 5: The structure of the agent design based on gRPC.

TABLE II: Parameters of the NMG test system.

\begin{tabular}{|l|c|c|c|}
\hline \multicolumn{4}{|c|}{ MG Level } \\
\hline DGs & $1-4$ & $5-7$ & $8-10$ \\
\hline $\begin{array}{l}K_{D G_{i}}^{P} \\
(\mathrm{~Hz} / \mathrm{kW})\end{array}$ & $4 \mathrm{e}-5$ & $3 \mathrm{e}-5$ & $2 \mathrm{e}-5$ \\
\hline $\begin{array}{l}K_{D G_{i}}^{Q} \\
\text { (p.u./kVar) }\end{array}$ & $1.2 \mathrm{e}-5$ & $0.9 \mathrm{e}-5$ & $0.6 \mathrm{e}-5$ \\
\hline $\begin{array}{l}\text { Load in } \\
\text { MG } k\end{array}$ & 1 & 2 & 6 \\
\hline$P$ (kW) & $15 \mathrm{e} 3$ & $15 \mathrm{e} 3$ & $15 \mathrm{e} 3$ \\
\hline$Q$ (kVar) & $8 \mathrm{e} 3$ & $10 \mathrm{e} 3$ & $6 \mathrm{e} 3$ \\
\hline \multicolumn{4}{|c|}{ NMG Level } \\
\hline MG $k$ & 1 & 2 & 6 \\
\hline $\begin{array}{l}K_{k}^{P} \\
\text { (Hz/kW) }\end{array}$ & $1 \mathrm{e}-5$ & $1 \mathrm{e}-5$ & $0.66 \mathrm{e}-5$ \\
\hline $\begin{array}{l}K_{k}^{Q} \\
\text { (p.u./kVar) }\end{array}$ & $3 \mathrm{e}-6$ & $3 \mathrm{e}-6$ & $2 \mathrm{e}-6$ \\
\hline Line & $1-4$ & $1-5$ & $2-4$ \\
\hline $\mathbf{Z}$ (p.u.) & $1.875+\mathrm{j} 1.228$ & $1.156+\mathrm{j} 0.491$ & $1.344+\mathrm{j} 0.969$ \\
\hline Line & $2-5$ & $3-5$ & $3-6$ \\
\hline $\mathbf{Z}$ (p.u.) & $0.781+\mathrm{j} 2.469$ & $1.625+\mathrm{j} 1.063$ & $1.875+\mathrm{j} 1.228$ \\
\hline
\end{tabular}

recording data from two sources is investigated: one is the logging files of the agents for checking the calculation in each iteration, and one is the measurement data saved in the simulator for observing system operation. There are five milestones we need to take into account: $t_{2}$ and $t_{4}$ when the disturbances occur in the system due to the changing of load power; $t_{1}, t_{3}$ and $t_{5}$ when the agents complete an ADMM loop and update new $\left\{P^{T e r}, Q^{T e r}\right\}$ to the lower control processes.

When a load step change in the NMG system, the objectives are summarized as follows:

- The primary control calculate the control inputs $\Omega_{M G_{k}}$ and $e_{M G_{k}}$ for MG level DG controllers in (3) and (4).

- In the secondary control level which has the response speed in seconds: the system frequency is restored to the nominal value of $50 \mathrm{~Hz}$; the PCC voltages of the NMG system (bus 1) will be restored to $1.00 p$.u, while the voltages at remain buses are guaranteed in the range of lower and upper threshold; the real and reactive power outputs from DGs are shared proportionally according to

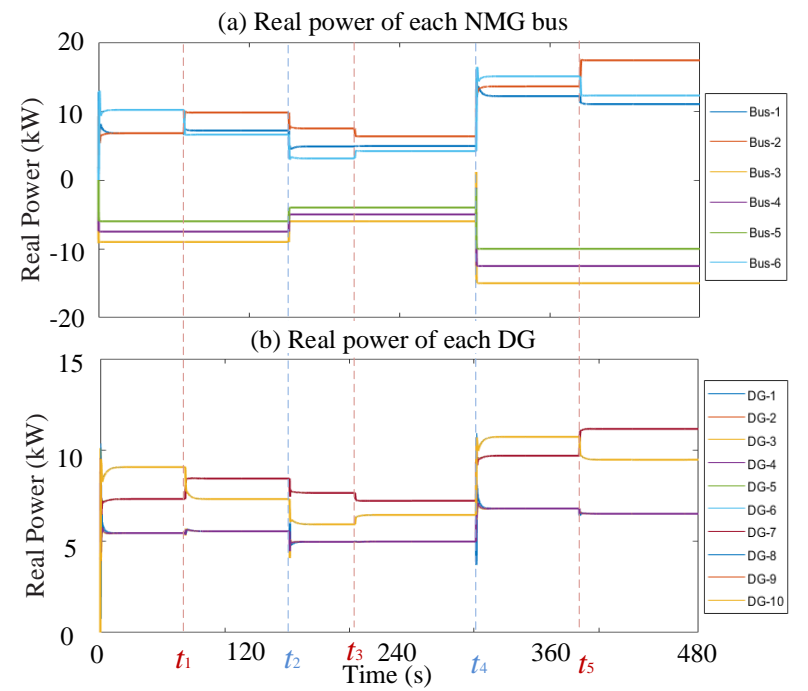

Fig. 6: Real power injection from each DG and bus in the NMG system.
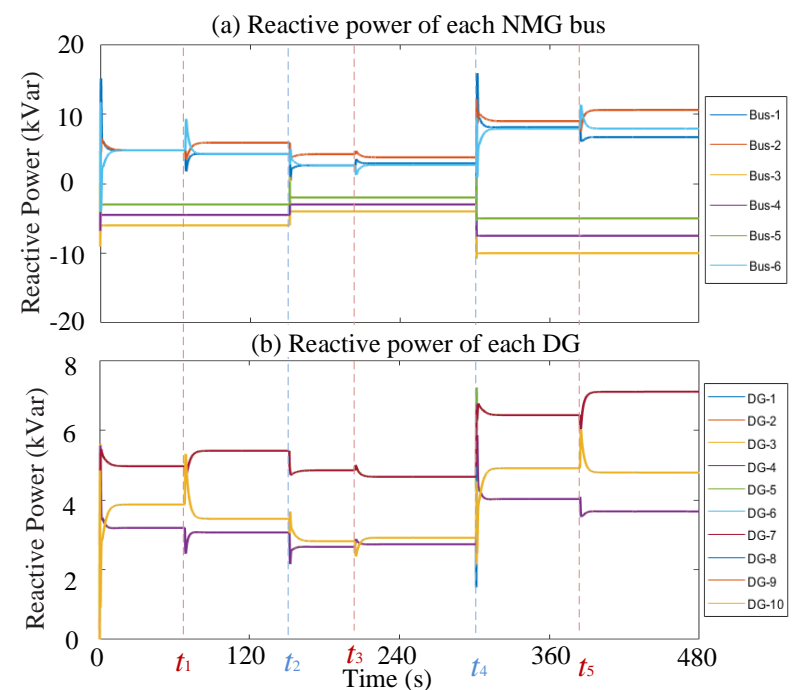

Fig. 7: Reactive power injection from each DG and bus in the NMG system.

the rated capacity.

- In the tertiary control level which has a slower dynamic response: the power outputs of MGs are redistributed to reduce total power losses to a minimal value.

Figs. 6-9 shows the real power, reactive power, frequency and bus voltages profiles during the HiL test. The results are illustrated in time sequence as follows:

1. $0 s \leq t<t_{2}$. The OPAL-RT starts at $t=0 s$. As shown in Fig. 6 (b) and 7 (b), the real and reactive power are shared among DGs in each MG are the same, according to the DG droop coefficients. The measured frequencies are restored to nominal values gradually as illustrated in Fig. 8. The PCC voltage at bus- 1 of the NMG is restored to 1p.u. Concurrently, the tertiary process executes the first ADMM loop when it gets the measurement and finishes the first loop at $t_{1}=67.9 \mathrm{~s}$. The optimal set-points for real and reactive power of each MG are sent to the secondary process. Then the dispatch order is executed to minimize the network power loss.

2. $t_{2} \leq t<t_{4}$. The load burden is decreased at $t_{2}=150 \mathrm{~s}$. 


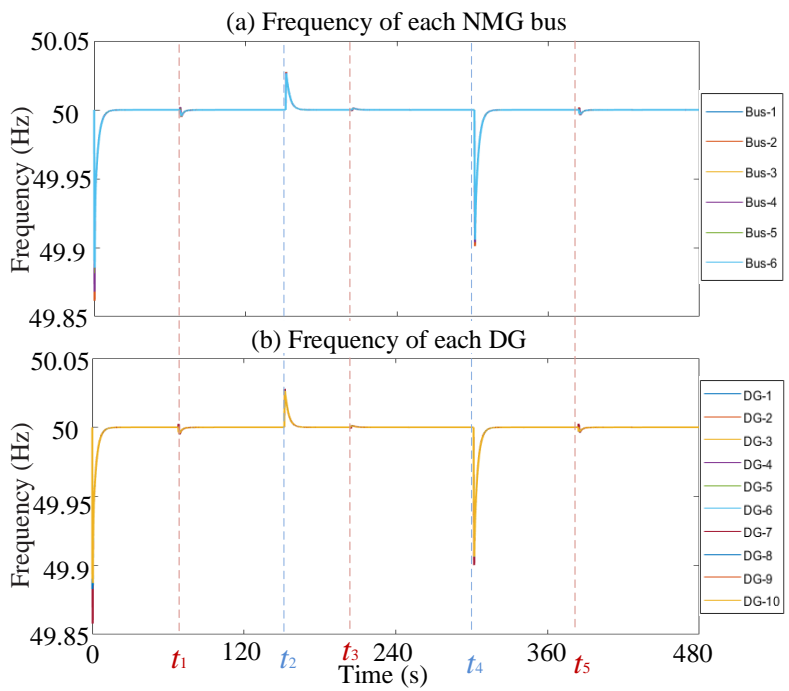

Fig. 8: The frequency profiles of each DG and bus in NMG.

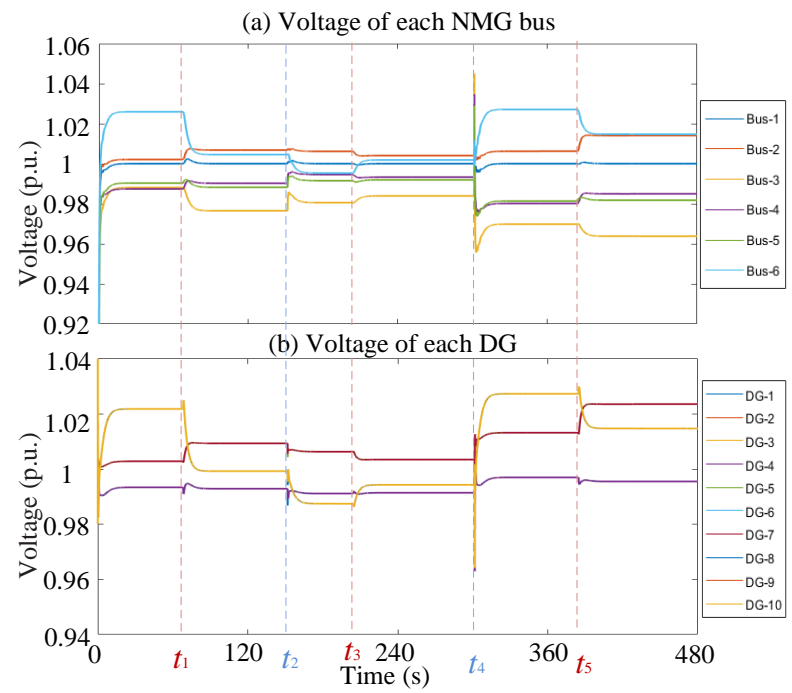

Fig. 9: The voltage profiles of each DG and bus in NMG.

The frequency and bus voltage suffer a sudden rise, but they can be rapidly restored to the references in time thanks to the activation of the secondary process in the agent. The power outputs among DGs in each MG keep the desired sharing ratios. After the load change, the ADMM process recognizes the system state change and updates the dispatch orders at $t_{3}=204.5 \mathrm{~s}$.

3. $t_{4} \leq t<480 \mathrm{~s}$. The load burden is increased at $t_{4}=$ $300 s$. The frequency and bus voltage suffer a sudden drop, but they can rapidly be restored to the references in time due to the secondary process in the agent. The power outputs among DGs in each MG keep the desired sharing ratios. After the load change, the ADMM process gets the measurement agent periodically and updates the dispatch orders at $t_{5}=384.8 \mathrm{~s}$.

As evidence, the processes in agent- 1 is further illustrated here. Figs. 10 (a) and 11 (a) show the logging files of calculated $\mathrm{P}$ and $\mathrm{Q}$ in ADMM process by the agent-1. Figs. 10 (b) and 11 (b) show the measured P and Q data from OPALRT for the comparison. From the logging files of the agents, we can observe that the ADMM process in each agent runs

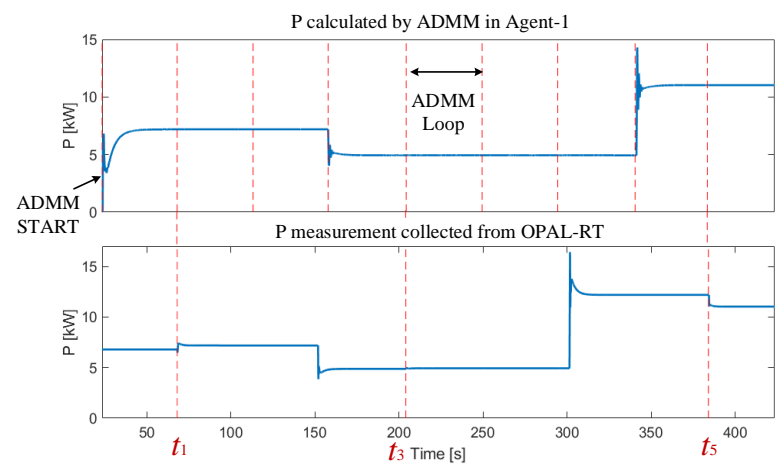

Fig. 10: Real power of MG-1 output calculated in Agent 1

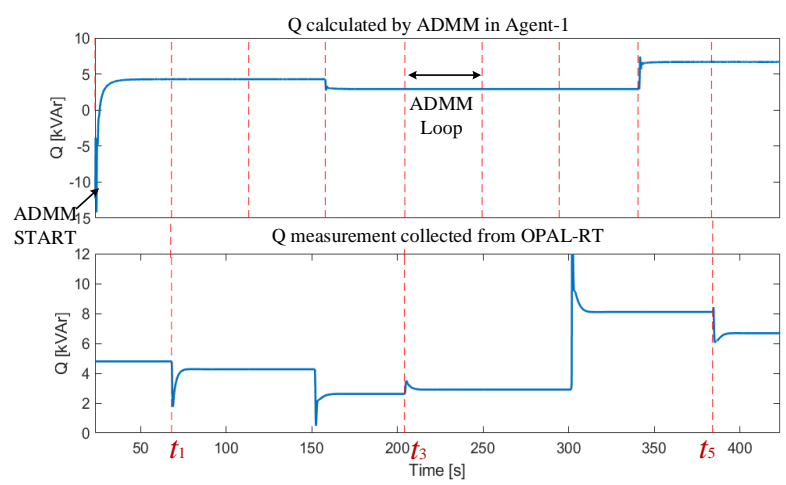

Fig. 11: Reactive power of MG-1 output calculated in Agent 1

9 ADMM loops, each loop consists of 1000 iterations. We investigate important milestones $t_{1}=67.9 \mathrm{~s}, t_{2}=204.5 \mathrm{~s}$ and $t_{3}=384.8 \mathrm{~s}$ in Figs. 10 and 11. The agent starts a few seconds after the OPAL-RT starts. At $t=23.4 s$, the ADMM process recognizes the system start. Then it takes $44.5 \mathrm{~s}$ to finish $l_{1}$ and calculate a pair of optimal set-points $P_{k}^{T e r}, Q_{k}^{T e r}$. The values are updated to the secondary process to minimize the network loss in the NMG. After a load change, the ADMM finished the third loops and start to calculate the system new set-points. Then the system operation points are updated again in $t_{2}=204.5 \mathrm{~s}$. Similarly process happened after load increase at $t_{4}=300 \mathrm{~s}$.

\section{Comparison Study}

In this section, the performance of the proposed control framework is further justified through comparison study.

Firstly, the proposed method is compared with typical secondary consensus control without ADMM loop, such as [16], [27]. The results of real power, reactive power, frequency and voltage at NMG level are shown in Fig. 12 (a)-(d), respectively. Comparing Fig. 12 (a) with Fig. 6 (a), the real power demand from the load bus 3, 4, 5 keeps the same. Similarly, as shown in Fig. 12 (b) and Fig. 7 (a), the reactive power demand from the load bus 3, 4, 5 are the same. In Fig 12 (a) and (b), the real and reactive power will only be shared according to the secondary control according to droop coefficients of each MG. Besides, as compared to Fig. 8 (a) and Fig. 9 (a), there will not be frequency and voltage variations when the ADMM loop results are dispatched to the secondary layer control.

Secondly, the total power losses of the MV network in the NMG are calculated and presented in Fig. 13. In Fig. 13 , the network power loss with the proposed method and 


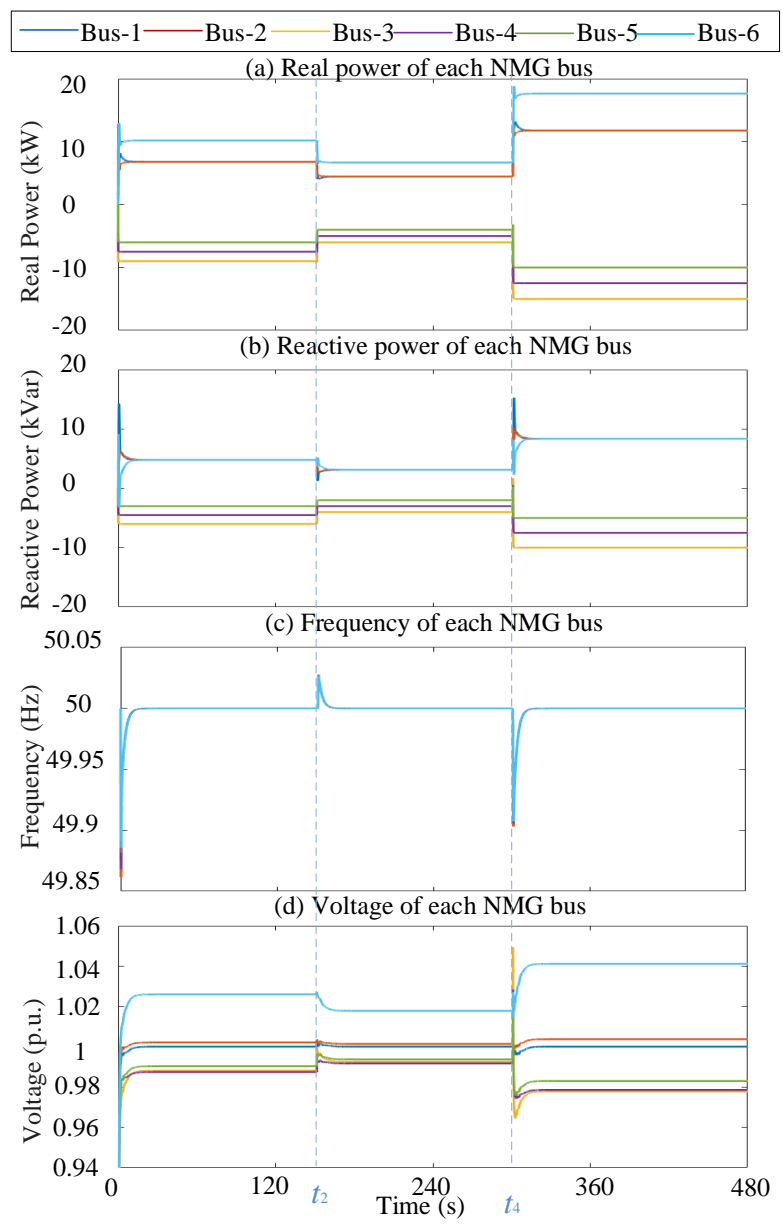

Fig. 12: System profiles without tertiary ADMM loops.

typical secondary consensus control excluding ADMM loop are compared. It can be observed clearly that the network power loss reduced when the control signals from ADMM process is adopted at $t_{1}=67.9 \mathrm{~s}$. The system optimal working points will be updated when there are load changes, and the optimal points will be re-dispatched at $t_{3}=204.5 \mathrm{~s}$ and $t_{5}=384.8 \mathrm{~s}$. After each update, the network power loss will be further reduced, as evidence from Fig. 13. The area, which indicates the accumulated energy loss in the NMG network, is also demonstrated.

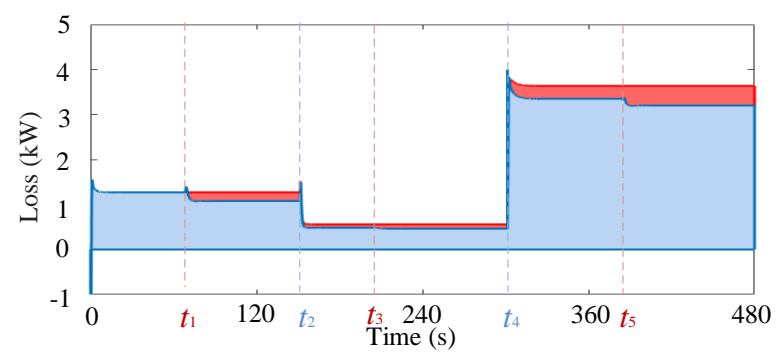

Fig. 13: The total power losses of the network.

Thirdly, to justify the correctness of tertiary control by ADMM, we have made a comparison with tertiary control results from the centralized optimization solved by Gurobi. The total power loss with the proposed method and the centralized method is compared in Fig. 14. It can be observed that network power loss with the ADMM loop will be the same as centralized optimization. The limitation of tertiary control based on ADMM is it takes longer time to be converged. However, the distributed P2P architecture will provide more flexibility, reliability, and scalablity, which gives a good option for NMG control system design.

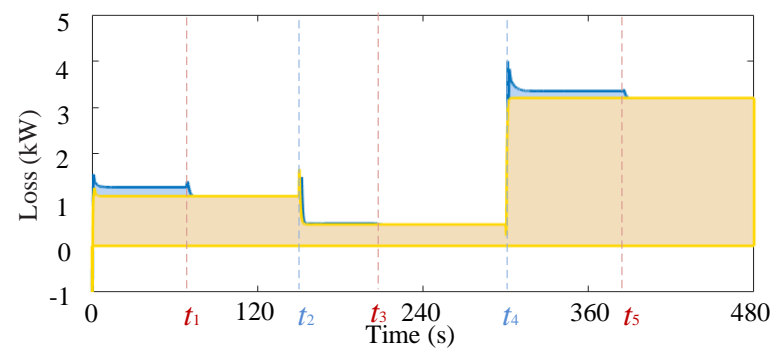

Fig. 14: The total real power losses of the network.

\section{CONCLUSION}

This paper presents a peer-to-peer control architecture for islanded NMG system. This P2P architecture is achieved by multi-layer and multi-agent algorithms and design, where multiple objectives are realized. The agent with capabilities of communication and computation can simultaneously run these processes corresponding to multi-layer controls. The NMG test system with six buses three MGs has been developed. The proposed control scheme has been validated on the platform as a practical approach. The results prove that the agents can work effectively in a environment close to working conditions. The major findings of this paper are summarized as follows:

- A novel P2P control architecture for NMGs is proposed with multi-agent and multi-layer design.

- A practical agent based realization is introduced considering the information exchange among agents and inter layers.

- The MAS HiL test proves the proposed control framework and agent design.

The future work includes: 1) distributed control scheme design for networked hybrid AC/DC NMGs; 2) cyber-resilient control design for NMG systems and hardware realization.

\section{REFERENCES}

[1] R. H. Lasseter, J. H. Eto, B. Schenkman, J. Stevens, H. Vollkommer, D. Klapp, E. Linton, H. Hurtado, and J. Roy, "Certs microgrid laboratory test bed," IEEE Transactions on Power Delivery, vol. 26, no. 1, pp. 325332, Jan 2011.

[2] D. E. Olivares, A. Mehrizi-Sani, A. H. Etemadi, C. A. Cañizares, R. Iravani, M. Kazerani, A. H. Hajimiragha, O. Gomis-Bellmunt, M. Saeedifard, R. Palma-Behnke, G. A. Jiménez-Estévez, and N. D. Hatziargyriou, "Trends in microgrid control," IEEE Transactions on Smart Grid, vol. 5, no. 4, pp. 1905-1919, 2014.

[3] J. M. Guerrero, M. Chandorkar, T. Lee, and P. C. Loh, "Advanced Control Architectures for Intelligent Microgrids; Part I: Decentralized and Hierarchical Control," IEEE Transactions on Industrial Electronics, vol. 60, no. 4, pp. 1254-1262, 2013.

[4] J. Rocabert, A. Luna, F. Blaabjerg, and P. Rodríguez, Control of Power Converters in AC Microgrids, Nov 2012, vol. 27, no. 11.

[5] F. Guo, C. Wen, J. Mao, and Y. D. Song, "Distributed Secondary Voltage and Frequency Restoration Control of Droop-Controlled Inverter-Based Microgrids," IEEE Transactions on Industrial Electronics, vol. 62, no. 7, pp. 4355-4364, 2015. 
[6] C. Zhang, Y. Xu, Z. Y. Dong, and J. Ma, "Robust operation of microgrids via two-stage coordinated energy storage and direct load control," IEEE Transactions on Power Systems, vol. 32, no. 4, pp. 2858-2868, July 2017.

[7] Z. Wang and J. Wang, "Self-healing resilient distribution systems based on sectionalization into microgrids," IEEE Transactions on Power Systems, vol. 30, no. 6, pp. 3139-3149, Nov 2015.

[8] Z. Li, M. Shahidehpour, F. Aminifar, A. Alabdulwahab, and Y. Al-Turki, "Networked microgrids for enhancing the power system resilience," Proceedings of the IEEE, vol. 105, no. 7, pp. 1289-1310, July 2017.

[9] M. N. Alam, S. Chakrabarti, and A. Ghosh, "Networked microgrids: State-of-the-art and future perspectives," IEEE Transactions on Industrial Informatics, vol. 15, no. 3, pp. 1238-1250, March 2019.

[10] Y. Wang, Y. Tang, Y. Xu, and Y. Xu, "A distributed control scheme of thermostatically controlled loads for the building-microgrid community," IEEE Transactions on Sustainable Energy, vol. 11, no. 1, pp. 350-360, 2020.

[11] S. Fang, Y. Wang, B. Gou, and Y. Xu, "Toward future green maritime transportation: An overview of seaport microgrids and all-electric ships," IEEE Transactions on Vehicular Technology, vol. 69, no. 1, pp. 207-219, 2020.

[12] X. Wu, X. Wu, Y. Xu, and J. He, "A hierarchical control framework for islanded multi-microgrid systems," in 2018 IEEE Power Energy Society General Meeting (PESGM), Aug 2018, pp. 1-5.

[13] X. Liu, P. Wang, and P. C. Loh, "A hybrid ac/dc microgrid and its coordination control," IEEE Transactions on Smart Grid, vol. 2, no. 2, pp. 278-286, June 2011.

[14] S. Su, Y. Yan, M. Jin, Q. Wang, and N. Zhou, "Hierarchical coordination control of multi-microgrids system in series and parallel structure," in 2012 China International Conference on Electricity Distribution, Sep. 2012, pp. 1-5.

[15] Q. Shafiee, T. Dragičević, J. C. Vasquez, and J. M. Guerrero, "Hierarchical control for multiple dc-microgrids clusters," IEEE Transactions on Energy Conversion, vol. 29, no. 4, pp. 922-933, Dec 2014.

[16] Z. Zhao, P. Yang, Y. Wang, Z. Xu, and J. M. Guerrero, "Dynamic characteristics analysis and stabilization of pv-based multiple microgrid clusters," IEEE Transactions on Smart Grid, vol. 10, no. 1, pp. 805-818, Jan 2019.

[17] Y. Wang, T. L. Nguyen, M. H. Syed, Y. Xu, V. H. Nguyen, E. GuilloSansano, G. Burt, Q. T. Tran, and R. Caire, "A distributed control scheme of microgrids in energy internet and its multi-site implementation," IEEE Transactions on Industrial Informatics, pp. 1-1, 2020.

[18] A. Hussain, V. Bui, and H. Kim, "A resilient and privacy-preserving energy management strategy for networked microgrids," IEEE Transactions on Smart Grid, vol. 9, no. 3, pp. 2127-2139, May 2018.

[19] A. Anvari-Moghaddam, A. Rahimi-Kian, M. Mirian, and J. Guerrero, "A multi-agent based energy management solution for integrated buildings and microgrid system," Applied Energy, vol. 203, pp. 41-56, Oct. 2017.

[20] Y. Li, T. Zhao, P. Wang, H. B. Gooi, L. Wu, Y. Liu, and J. Ye, "Optimal operation of multimicrogrids via cooperative energy and reserve scheduling," IEEE Transactions on Industrial Informatics, vol. 14, no. 8, pp. 3459-3468, Aug 2018.

[21] N. Bazmohammadi, A. Tahsiri, A. Anvari-Moghaddam, and J. M. Guerrero, "Stochastic predictive control of multi-microgrid systems," IEEE Transactions on Industry Applications, vol. 55, no. 5, pp. 53115319, Sep. 2019.

[22] N. Bazmohammadi, A. Tahsiri, A. Anvari-Moghaddam, and J. Guerrero, "A hierarchical energy management strategy for interconnected microgrids considering uncertainty," International Journal of Electrical Power \& Energy Systems, vol. 109, pp. 597-608, Jul. 2019.

[23] S. Yao, P. Wang, and T. Zhao, "Transportable energy storage for more resilient distribution systems with multiple microgrids," IEEE Transactions on Smart Grid, vol. 10, no. 3, pp. 3331-3341, May 2019.

[24] Y. Liu, Y. Li, H. Xin, H. B. Gooi, and J. Pan, "Distributed optimal tie-line power flow control for multiple interconnected ac microgrids," IEEE Transactions on Power Systems, vol. 34, no. 3, pp. 1869-1880, May 2019.

[25] Y. Li, P. Dong, M. Liu, and G. Yang, "A distributed coordination control based on finite-time consensus algorithm for a cluster of dc microgrids," IEEE Transactions on Power Systems, vol. 34, no. 3, pp. 2205-2215, May 2019.

[26] W. Zhang and Y. Xu, "Distributed optimal control for multiple microgrids in a distribution network," IEEE Transactions on Smart Grid vol. 10, no. 4, pp. 3765-3779, July 2019.

[27] Y. Wang, T. L. Nguyen, Y. Xu, Z. Li, Q. Tran, and R. Caire, "Cyberphysical design and implementation of distributed event-triggered sec- ondary control in islanded microgrids," IEEE Transactions on Industry Applications, vol. 55, no. 6, pp. 5631-5642, Nov.-Dec. 2019.

[28] A. Bidram, F. L. Lewis, and A. Davoudi, "Distributed control systems for small-scale power networks: Using multiagent cooperative control theory," IEEE Control Systems, 2014.

[29] J. E. S. Boyd, N. Parikh, E. Chu, B. Peleato, "Distributed Optimization and Statistical Learning via the Alternating Direction Method of Multipliers," Foundations and Trends in Machine Learning, 2011.

Yu Wang (S'12-M'17) received the B.Eng. degree in School of Electrical Engineering and Automation from Wuhan University, China in 2011, and the M.Sc. and Ph.D. degree from Nanyang Technological University, Singapore in 2012 and 2017, respectively. He is currently a research fellow in Nanyang Technological University, Singapore. He is investigating industry projects on hybrid microgrid systems, energy storage systems, and cyber-security of power systems. His research interests include distributed control and optimization in electrical systems, microgrids and cyber-physical systems.

Tung-Lam Nguyen received the Master's degree in electrical engineering from National Taiwan University of Science and Technology, Taiwan, in 2014, and the Ph.D. degree from the University of Grenoble Alpes, Grenoble, France, in 2019. He is currently a Lecturer with the Faculty of Electrical Engineering, The University of Danang, University of Science and Technology, Danang, Vietnam, and a Post-Doctoral Researcher with the Grenoble Institute of Technology, Grenoble, France, as part of the G2ELab. His research interests include distributed control and optimization in microgrids and distribution networks, and co-simulation in smart grid.

Yan Xu (S'10-M'13) received the B.E. and M.E degrees from South China University of Technology, Guangzhou, China in 2008 and 2011, respectively, and the Ph.D. degree from The University of Newcastle, Australia, in 2013. He is now the Nanyang Assistant Professor at School of Electrical and Electronic Engineering, Nanyang Technological University (NTU), and a Cluster Director at Energy Research Institute@ NTU (ERI@N), Singapore. Previously, he held The University of Sydney Postdoctoral Fellowship in Australia. His research interests include power system stability and control, microgrid, and data-analytics for smart grid applications. $\mathrm{Dr} \mathrm{Xu}$ is an Editor for IEEE Transactions on Smart Grid, IEEE Transactions on Power Systems, IEEE Power Engineering Letters, CSEE Journal of Power and Energy Systems, and an Associate Editor for IET Generation, Transmission \& Distribution.

Quoc-Tuan Tran received his Ph.D. degree in Electrical Engineering and Habilitation à Diriger des Recherches degree from the Grenoble Institute of Technology in 1993 and 2000, respectively. He is an IEEE senior member. He is currently a Professor with INSTN - Paris Saclay University, and a Scientific Manager with Alternative Energies and Atomic Energy Commission (CEA) - National Institute for Solar Energy (INES). His research interests are in the fields of power system analysis, operations, electromagnetic transients, distributed generation, smart-grid and renewable energy.

Raphael Caire received the Diplôme d'Etudes Approfondies and Doctorat de l'INPG degrees from the Institut National Polytechnique de Grenoble in 2000 and 2004, respectively. He has been with the Power Electronics field at the Center of Power Electronic System (CPES), USA, since 2000 and within several EDF research centers in Germany and France from 2004 to 2006. He is currently an Associate Professor with the Grenoble Institute of Technology, Ecole d'ingénieurs en énergie eau et environnement, Grenoble Electrical Engineering Laboratory. His research is centered on the impacts, production control of dispersed generation on distribution system and critical infrastructures. 